Cahiers

Claude Simon

\section{Cahiers Claude Simon}

15 | 2020

« je pouvais voir»

\title{
Le monument aux morts de Claude Simon
}

claude Simon's War Memorial

\section{Aymeric Glacet}

\section{OpenEdition}

\section{Journals}

Édition électronique

URL : https://journals.openedition.org/ccs/2928

DOI : $10.4000 /$ ccs. 2928

ISSN : 2558-782X

Éditeur :

Presses universitaires de Rennes, Association des lecteurs de Claude Simon

\section{Édition imprimée}

Date de publication : 3 septembre 2020

Pagination : 141-154

ISBN : 978-2-7535-8065-7

ISSN : 1774-9425

\section{Référence électronique}

Aymeric Glacet, « Le monument aux morts de Claude Simon », Cahiers Claude Simon [En ligne], 15 | 2020, mis en ligne le 03 septembre 2021, consulté le 05 décembre 2022. URL : http:// journals.openedition.org/ccs/2928; DOI : https://doi.org/10.4000/ccs.2928 


\title{
LE MONUMENT AUX MORTS DE CLAUDE SIMON
}

\author{
Aymeric GLACET
}

Le Tramway, c'est le TRANSIT.

C'est par l'écriture de ce dernier roman que Claude Simon passe de l'autre côté du miroir. Puisque transir, à l'origine, c'est mourir. Et quelle plus belle mort que celle que l'on choisit? Quelle plus belle mort, pour un écrivain, que de se la raconter pour mieux la tromper? Être en transit, c'est attendre la mort comme l'attendait Claude Simon envoyé " aux Urgences " (Tram., p. 1260) et incertain d'en revenir. Lorsque le mot apparaît dans le texte pour la première fois, c'est d'ailleurs tronqué comme le sont les "hommes-troncs " (p. 1257) rescapés de la guerre : ce n'est pas le mot TRANSIT que l'on peut lire alors, mais « RANS » (p. 1260) comme sont rances les corps du père, de la mère et de l'écrivain lui-même.

Le Tramway sent le rance, en effet, non seulement " l'étroite et prestigieuse cabine " (p. 1256) du wattman, avec ses " relents ammoniacaux » (p. 1261) qui rappellent au narrateur les "effroyables émanations des latrines d'urgence " (p. 1256) des camps de concentration, mais encore le livre lui-même dans lequel le père n'en finit pas de se décomposer au pied d'un arbre parce que sa femme n'aura jamais retrouvé son corps et que son fils ne pourra l'enterrer qu'entre les pages de son œuvre; livre dans lequel la mère se décompose malgré le régime qu'un " "ponte" de la chirurgie" (p. 1266) lui a prescrit pour lutter contre le cancer qui la ronge et la condamne à sa " liseuse " (mot sur lequel l'écrivain revient avec insistance parce qu'il regrette qu'elle n'ait jamais pu le lire) qui va bientôt être " jetée au pourrissoir " (p. 1282) où finissent les rats que la bonne "brûlait vivants » et les chatons qu' elle tuait « en les jetant avec violence contre le mur» (p. 1294); livre dans lequel le fils est hospitalisé face à « une sorte de 
double ricanant " (p. 1278) de lui-même et à la "déchéance " (ibid.) d'un corps « rendu impropre à vivre " (p. 1279), un corps dont le contact imprègne des serviettes-éponges aux " évanescentes couleurs moisies » qui n'étaient pas de couleur rose, "mais comme un vieillissement, une sorte de sénilité du rouge " $($ ibid $)$.

La vie en rose, par conséquent. Ou ce qu’il en reste, comme si le rose était le fil conducteur de ce dernier roman, une couleur à laquelle l'écrivain ne nous avait pourtant pas habitués, lui qui aime tant le jaune, mais une couleur à laquelle, avec l'âge, il semble ne plus pouvoir échapper. Car de la même façon que tout jaunit (ainsi les visages de la mère et de sa bonne), tout finit par virer au rose. À moins que ce ne soit le rose qui vire, tant il semble la couleur du transit par excellence, une couleur qui oscille entre " foetus » et « squelettes » (p. 1274), entre le " rose vif " (p. 1284) de la vie et le " corps livide » (p. 1288) de la mort, et qui nous brancarde ainsi « du service d'obstétrique à la morgue, offrant comme en raccourci (ou en condensé) les successifs états de la machine humaine de la naissance à l'agonie en passant par toutes les déviations et anomalies possibles jusqu'à sa définitive corruption " (p. 1299).

De fait, le RANS est au TRANSIT ce que le corrompu est au transitoire, le mort au vivant, l'image fixe à l'image en mouvement, Claude Simon cherchant à toute fin, dans ce "roman testamentaire ", comme le définit Mireille Calle-Gruber, à faire la paix avec lui-même et avec tous ceux que son œuvre a exhumés avant que le rideau ne tombe une dernière fois comme " un uniforme linceul" (p. 1317). Et pour y parvenir, faire son mea culpa. On pourrait ainsi faire grand cas de cet écrivain qui condamne la corruption des élites politiques détruisant peu à peu la ville historique de son enfance à coups de pots-de-vin tout en reconnaissant qu'il est un aristocrate qui héritera de vignobles produisant un vin de dessert revendu à Cinzano pour "vivre sinon luxueusement, tout au moins dans l'aisance » (p. 1292), un patrimoine qui l'obligea sans doute à se demander quelles sont les conditions nécessaires pour être un artiste à part entière, et qui le força, pour s'en justifier, à faire de la guerre, puisqu' elle n'épargnait alors aucune classe sociale, la cause première responsable de l'anéantissement de sa famille.

C'est alors d'une autre corruption qu'il s'agit, celle par exemple qui pousse un écrivain au sommet de son art à écrire un roman qui ressemble assez peu, finalement, à ceux qui l'ont précédé comme s’il avait cherché à écrire un mode d'emploi accessible à tous pour expliquer d'où il vient et quel a été son trajet. Sans faire de mauvais jeu de mots, Le Tramway a des airs de roman de 
gare, un de ces romans qu'on pourrait lire dans le métro le matin en allant au boulot. C'est un petit livre, facilement transportable, facile à lire. Le Tramway condense toute l'œuvre en quelques pages et en précipite tous les thèmes, une sorte de carte routière ou d'almanach des chemins de fer, et c'est en cela précisément qu'il est remarquable.

Que faire d'un texte, en effet, qui décode le projet romanesque et semble nous en donner la clé comme si l'écrivain, assis à nos côtés sur la banquette du Tramway, nous montrait par la fenêtre les différentes étapes de son travail pour remonter l'œuvre comme on remonte le temps? Le titre est à ce titre révélateur qui est un sous-titre en lui-même. Quand on sait l'importance des sous-titres pour Claude Simon, on peut être surpris par ce titre en rupture avec les précédents. Nous sommes le 15 mars 2001 et, contre toute attente, après Histoire, après La Bataille de Pharsale, après L'Acacia et Le Jardin des Plantes, c'est ce drôle de petit mot qui fait son entrée sur la scène littéraire.

Et Claude Simon, dès la deuxième page, de s'en excuser lorsqu’à propos de l'inscription "Défense de parler au wattman », il se moque du "franco-anglais approximatif» (p. 1253) du mot employé. Comment ne pas penser qu'il s'agit alors, la situation s'y prêtant tout particulièrement, de l'hôpital se moquant de la Charité? Si wattman (alors que traminot, mot-valise fait sur le modèle de cheminot, était en usage à Marseille), si wattman est du mauvais franglais, c'est-à-dire un faux anglicisme qui désigne d'abord le conducteur d'un véhicule automobile avant de désigner le conducteur d'un "trolley " (p. 1283) électrique, que dire de tramway qui, en français, ne désigne pas la voie, comme en anglais, mais la voiture, qui devrait plutôt se dire tramway car, tramcar, ou encore, comme en Amérique, streetcar, et, à San Francisco, cable car, enfin tout sauf tramway, même si c'est ainsi, après tout, que les langues, profitant de l'ignorance de ceux qui n'en parlent qu'une, se réinventent.

C'est en cela finalement que ce titre est si peu simonien puisqu'il est si impropre. Ou qu'il l'est, propre, plus que tout autre, non pas parce qu'il est typiquement français, mais parce qu'il est lui-même en transit entre deux mondes, deux époques, deux guerres, deux terminus, entre père et mère, cinéma et photographie, qu'il est, tout simplement, l'entre-deux où tous les fils se nouent. Car le tramway, ou plutôt "le tram " (p. 1265), si l'on ne considère, avec Claude Simon, que l'apoconyme, c'est aussi la trame, c'est-àdire, à l'origine, les rails en bois sur lesquels circulaient les chariots des mines de charbon. 
Mais cette trame, bien évidemment, ce n'est pas seulement «l'anarchique tissu urbain " (p. 1299), c'est aussi la trame de l'œuvre, la trame de la vie, l'intrigue ourdie, les déviations et les anomalies, les "infimes coïncidences " (prière d'insérer) qui amènent les trajets à se confondre, le nœud gordien et les parenthèses d'Orion aveugle, lorsque le « sentier " de l'écrivain " tourne et retourne sur lui-même, comme peut le faire un voyageur égaré dans une forêt, revenant sur ses pas, repartant, trompé (ou guidé?)»( $E I$, p. 1183). Comme souvent, et proustienne en cela, se trouve entre parenthèses quelque vérité à lire. L'écrivain, s'il se tourne et se retourne dans son œuvre comme dans son lit d'hôpital, n'étant pas tant trompé que guidé. Et le guide n'est autre que ce tramway dont "les allées et venues d'un terminus à l'autre entre les ondulations des vignes ponctuent le cours des vies" (prière d'insérer) comme ils ponctuent les phrases du roman pour faire de l'écriture une réalité à part entière.

Sauf qu'en l'occurrence, guidé, il l'est par un mot qui n'en est pas vraiment un, un mot qui a le cul entre deux langues et dont Alain Rey ajoute qu'en anglais, il est composé de tram «brancard ", se trouvant ainsi apparenté à branche, et que la locution figurée "ruer dans les brancards " tire son origine d'une pièce de bois prolongeant la caisse d'une voiture et permettant d'atteler un cheval. Voilà que par analogie d'abord, et par métonymie ensuite, de la liseuse de maman au "brancard " (p. 1306) des processions religieuses, on en arrive à la " civière " de Claude Simon en TRANSIT aux Urgences alors qu'un ivrogne passe devant lui " galopant" (p. 1262) comme un cheval et le tire de son engourdissement avant de le replonger dans un autre temps et un autre lieu où son œuvre finit toujours par le ramener, c'est-à-dire un 10 mai alors qu’il traverse un "hameau au joli nom émaillé de fleurs" (p. 1312).

On pourrait continuer ainsi longtemps, et expliquer comment le mot branche, du latin branca "patte d'un animal ", créé par analogie de forme, a supplanté l'ancien français ram, représentant du latin ramus, de rameau. Et dès lors, des " rameaux » (p. 1292) de L'Acacia à la trameuse en photogravure en passant par la navette du tisserand ${ }^{1}$, filer la métaphore que Claude

1. Trame est la réfection (1549) d'après tramer de traime (XII ${ }^{\mathrm{e}}$ siècle) et treme (v. 1150), employé jusqu'au $\mathrm{XVIII}^{\mathrm{e}}$ siècle par les tisserands, issus du latin trama, mot technique attesté depuis Varron qui désigne les fils de la chaîne quand, séparés par les lices, ils livrent passage à la navette puis, par extension, la chaîne de tissu ou la trame. Il est issu de trans «à travers ", ce qui fait que tramway et transit sont apparentés pour désigner l'ensemble des fils passés entre les fils de la chaîne pour faire un tissu et qui par conséquent a développé des sens figurés comme "vie " (1636), ainsi la "trame de la vie ", à l'époque classique, ou " intrigue élaborée pour nuire ", au début du XVII e siècle, notamment en littérature. D'où tramer, ourdir un complot, d'où le trameur, le tisserand, et la trameuse, en photogravure. 
Simon n'hésite pas à dévider, jouant de toute cette ramification à l'œuvre dans l'œuvre, de tous ces branchements électriques des mots les uns avec les autres jusqu'à ce que tout le circuit soit mis sous tension et illumine l'œuvre aussi sûrement qu'un panneau publicitaire. Mais passons pour le moment, et remarquons seulement que ce panneau publicitaire est rose. Aussi rose que ce visage de femme paraissant " sans vie " et pourtant " rose ", ou " rosi par la lumière " (p. 1260) "ce masque flou et rose absolument inexpressif " (ibid.) qu'aperçoit le narrateur lorsqu'il est en TRANSIT sur son lit roulant alors qu'elle-même gît non pas sur ce qu'on pourrait "appeler un lit roulant» (p. 1305) mais une "impressionnante machine faite de tubes d'acier» (ibid.) qui est "guidée " (ibid.) par des infirmiers comme si elle était sur des rails de tramway aux allures de corbillard, sa tête " encore rose et fraîche " (ibid.) dépassant seule de la couverture et ne laissant aucunement présager " quelque effroyable maladie" (ibid.) dont on ne se remet pas. Guidée vers la mort, comme le narrateur craint de l'être, qui est lui-même relié à tout un "appareillage compliqué de tuyaux " (p. 1308), elle l'est donc en dépit de la fraîcheur de son teint.

C'est que le rose, contre toute apparence, est trompeur, qu'il masque la mort, qu'il est même, tout bien considéré, avec toutes ses nuances qui vont du lilas au mauve et de la prune à l'ocre foncé en passant par le «brun-rouge sale» (p. 1310), la couleur même de la mort. Et lorsqu'entre les pages elle sort le bout de son nez, ce n'est jamais de bon augure. Le rose est toujours trop rose pour être honnête : "La malade au visage trop rose et flou " (p. 1261) échappe au " monde des vivants " (ibid.) et disparaît dans le couloir de la mort qui lui aussi rappelle le "vestibule aux relents ammoniacaux " (ibid.) des camps de concentration. D'ailleurs, le pouvoir de ce rose flou et énigmatique est tel qu'il condamne l'observateur à une " espèce de vase, de brouillard sans avant ni après » (ibid.), cet "impalpable et protecteur brouillard de la mémoire " (p. 1317) sur lequel se clôt non seulement le roman, mais encore l'œuvre et la vie de son auteur.

Comprendre alors en quoi ce brouillard de la mémoire, cette condensation du temps sans passé et sans avenir (" sans avant ni après » est l’une des expressions qui revient constamment sous la plume de l'auteur), est protecteur, et pour le comprendre revenir rapidement aux deux citations mises en exergue. Se douter que Claude Simon les a choisies et mises dans cet ordre afin qu'elles se complètent et enrichissent ce qui suit, et en conclure que le sens est au mot ce que la vapeur est à la lumière. Si le sens est comme une vapeur extérieure à 
l'objet qu'il cherche à définir, c'est qu'il est, selon la citation de Conrad, une explication possible à laquelle l'objet en question ne saurait se limiter tout à fait mais derrière laquelle il se dissimule par défaut. Si on considère que cet objet est une image, "le seul élément essentiel » selon la citation de Proust, alors cette image n'a de sens que pour celui qui la voit et ce qu'il en discerne à un moment donné. Si le brouillard est à la mémoire ce que la vapeur est au sens, l'image est isolée du reste du monde par le sens qu'on lui donne tout comme la mémoire ne se rapporte plus qu'à ce que les phares d'un véhicule éclairent lorsqu'ils cherchent à le couper au couteau, c'est-à-dire " une sorte d'entonnoir, d'insondable et noire perspective» (p. 1263).

Il y a toujours un voile entre le vécu et le souvenir qu'on en garde, et « ce voile en suspension» (p. 1317), comparable à " un uniforme linceul » (ibid.) ou à " une impalpable couche de cendres " (ibid.), sent le brûlé, le croupi et, de l'ancien provençal ransa, le fané. Claude Simon a beau mettre cela sur le compte de la fatigue ou de la fièvre, il sait bien que la maladie tout comme le trajet en ambulance sont une métaphore de la vie que l'on se contente « d'enregistrer confusément " (p. 1308) et que l'écriture a pour but de mettre en lumière et de réaliser. Dès lors, le flou rose de la mémoire est protecteur non pas parce que la vérité aveugle comme la «lumière aveuglante » (p. 1291) du soleil ou parce que le brouillon qu'est la mémoire permet de se voiler la face, mais parce qu'elle est un écran sur lequel projeter les images défraîchies d'un passé que l'écriture présente à nouveau.

Le présent de l'écriture, en effet, ce fameux présent simonien, doit se comprendre dans le contexte d'une mémoire qui agit comme l'écran-filtre de la photogravure. La mémoire est passée à travers un écran coloré en rose qui la pétrifie comme un voile de statue. Linceul fossilisé (Claude Simon avait conscience qu'il tirait sa révérence avec Le Tramway et qu'il s'agissait sans doute de son ultime représentation), l'écriture lui donne la vie éternelle. Et si le rose est amer, c'est parce que cette suite d'images sans relations de cause à effet n'a pas pour vocation d'embellir les souvenirs par un travail de l'imagination ou d'en débrouiller les fils. Pas question d'inventer des coïncidences hasardeuses qui donneraient à tout ce charivari un semblant de légitimité dépendant de circonstances aussi éphémères que "l'Histoire " (p. 1286) ou " le Progrès » (p. 1287). Au contraire, l'écriture a ce privilège, selon Claude Simon, de sculpter sa propre réalité. Raison pour laquelle l'auteur doute constamment de sa mémoire comme s'il voulait s'assurer qu'il n'avait pas corrompu d'affabulations ce dont il se souvenait : "si je me souviens bien » 
(p. 1283 et 1299) ou " je ne sais plus " (p. 1285) ou « je ne me souviens pas » (p. 1296), se plaît-il ainsi à répéter. Et tel serait tout l'intérêt du tramway, qui est guidé d'une main de maître, droit sur ses rails, sans possibilité de s'égarer dans la forêt des sens, la présentation de l'œuvre enfin réduite à une seule voie ferrée, qui fend le brouillard sans s'imaginer le disperser, jusqu'au cour de la trame où, comme de bien entendu, on retombe en enfance parce que c'est là, après tout, que tout commence et tout finit.

On ne connaît réellement quelqu'un que si l'on connaît son enfance, c'est ce que l'écrivain réalise à la veille de mourir, et c'est, revenant dans son dernier roman sur les premières années de sa vie à Perpignan, ce qu'il tient, plus que tout, à dire. Ne pas s'étonner alors que la première fois que le mot rose apparaît dans ce " cortège [de] souvenirs enfantins " (p. 1313), c'est à propos des billets « de couleurs pastel» (p. 1257), et notamment roses, qui permettent de prendre le tramway, ce "fatidique tramway" (p. 1284) qui est à l'enfant ce que la barque de Charon est à l'adulte, la ligne de fuite qui fait passer des "pimpantes tuiles rose " (p. 1301) qui plaisent tant aux touristes, aux murs moisis "badigeonnés d'un rose presque décoloré " virant " au mauve " (p. 1271) de la maison de "madame Espinosa ", "relation de plage " de maman, " jamais invitée aux soirées musicales ou aux bridges " (p. 1270) bien que " la question de la judéité [n’ait] jamais été évoquée " (Ibid.), se défend l'auteur, et pour cause, ce nom, Espinosa, étant aussi celui de Spinoza, Baruch Spinoza, juif portugais d'Amsterdam, Marrane excommunié comme l'était par maman la mère du petit Gaguy.

C'est en effet par l'intermédiaire de son ami d'enfance lui aussi disparu à la guerre que Claude Simon, dans le même passage, semble inviter Spinoza à répondre directement à Proust critiqué pour son invraisemblance ainsi que son recours à la contingence et à la nécessité d'un enchaînement de causes nous déterminant et définissant notre liberté. Recourant "sans vergogne aux procédés les plus éculés (comme les hasards, les coïncidences, le fortuit) 》 (p. 1276), Proust en revient à écrire "involontairement " (ibid.) et s'oppose au conatus spinoziste. Pour Claude Simon, qui l'accuse de faire des choix narratifs arbitraires, la transcendance est dans l'immanence. Tout être fini est une détermination de l'infini et obéit nécessairement aux lois divines ou naturelles, à celles de la vie ou de l'énergie cosmique, peu importe d'ailleurs la façon dont on les nomme. La seule véritable liberté se gagne grâce à l'énergie créatrice intérieure. C'est dans le moment de l'écriture, dans son imminence instantanée, que l'être simonien se dépasse. Et c'est en refusant de lâcher le 
mot de Dieu ou de hasard, c'est en jouant au jeu du pourquoi des enfants tout en sachant qu'on ne peut pas régresser à l'infini, c'est en remontant à l'enfance (lorsqu'il avait, entre cinq ans et onze ans, l'âge de l'éternel présent) comme à la cause première, qu'il parvient à la raison littéraire.

Refusant de répondre à l'ultime pourquoi, considérant sans doute que cette réponse est l'asile de l'ignorance puisque le mutisme de Dieu est la preuve que le seul absolu auquel l'homme puisse aspirer est l'art qu'il produit, Claude Simon est étranger à une mémoire qui serait le fruit du hasard au point que s'il intervient dans le Tramway, ce n'est jamais par hasard. C'est au contraire parce qu'il a réussi à attraper le tram lorsque « le hasard faisait coïncider l'humeur du professeur et celle du wattman » (p. 1266). Ce qui n'arrive presque jamais. Et pour cause : il a beau se plaindre que tout se ligue contre lui pour qu'il le rate à la sortie de l'école, ce qui d'ailleurs l'arrange bien², il explique par ailleurs que "deux tramways partis chaque heure en même temps se croisaient à mi-parcours » (p. 1255), raison pour laquelle celui qu'il devait prendre en face du cinéma ne pouvait retarder son départ serait-ce de cinq minutes, parce qu'alors il ne se serait pas trouvé au bon endroit au bon moment pour croiser celui arrivant en sens inverse au niveau du " "garage" (nom que l'on donnait au dédoublement des voies qui, à mi-parcours, permettait aux deux motrices de se croiser) » (p. 1255-1256) et qui n'était autre qu'une sorte de gare de transit.

Pas question, autrement dit, que le hasard fasse bien les choses. On ne peut pas lui faire confiance puisque d'un danger - cinéma, jeu des acteurs, femmes sensuelles et montagnes russes - à l'autre - plage mondaine à la mauvaise réputation ou "sommaire cube de bêton » (p. 1268), c'est-à-dire casino et jeux d'argent en passant par Gaguy, le « compagnon de jeux » dont le nom est lui-même un gag parce que sa mère Espinosa est une " médiocre joueuse de bridge » (p. 1270) -, jamais un coup de dés ne saurait l'abolir. Aussi son écriture ressemble-t-elle plutôt à une partie d'échecs dans laquelle aucun mouvement n'est le fruit du hasard.

Remarquons alors que Spinoza, qui menait une existence d'ascète, qui meurt dans une grande pauvreté, sans sépulture, ne laissant derrière lui que sa bibliothèque et un petit jeu d'échecs noué dans un sachet, signait ses lettres

2. «Lorsque, par chance, je ne manquais pas le tramway de 4 heures (c'est-à-dire quand le hasard faisait coïncider l'humeur du professeur et celle du wattman) [...] l'amour maternel s'étant chez elle peu à peu empreint [...] d'une sorte de violence désespérée [...] elle s'était muée en un personnage d'une dureté rigide qui, chaque jour, me faisait appréhender le moment de la retrouver» (p. 1266-1267). 
avec une chevalière dont le sceau représentait une rose sous laquelle se trouvait l'inscription latine caute, méfie-toi. Et, en effet, de la rose se méfier des épines, que l'on retrouve dans le portugais espinoza, se méfier de la fleur donc, de celle offerte à la tête rose et reposant désormais " à la morgue " (p. 1304), mais aussi de son homonyme. Car le rose, dans Le Tramway, plus que n'importe quel autre mot, faisant des allers-retours entre la vie et la mort ${ }^{3}$ et suscitant les associations, fait correspondre entre eux les différents éléments nécessaires à la trame romanesque, c'est-à-dire à l'écriture ${ }^{4}$. D'ailleurs, à peine le mot avait-il été lâché que le "monument aux morts » faisait son apparition.

Et quel monument!

Quel «monumental monument» (p. 1257) autour duquel se retrouvent les hommes-troncs que la mère du narrateur appelle ainsi avec " une sorte de joie mauvaise " (ibid.) et, tout compte fait, à tort, puisqu'il s'agit de culsde-jatte, Claude Simon précisant sans pour autant la corriger que seul « l'un d'eux était de surcroît manchot » (p. 1259), ce qui en fait le seul véritable homme-tronc de la bande. Et sans doute la joie mauvaise de la mère n'a d'égal que son envie et sa jalousie puisqu'elle les déteste, ces rescapés qui lui rappellent la mort de son époux et, " comme par une sorte de mimétisme " (p. 1259), sa propre mort à venir, elle qui partage avec ces vétérans le « même visage osseux et dur de rapace " (p. 1257), et comme eux est condamnée à sa liseuse à roulettes comme ils sont condamnés à leurs "voiturettes " (ibid.) à trois roues, Claude Simon finissant par nous dire qu'elle faisait « irrésistiblement penser à la fin, en féminisé, terreux et impitoyable, à ceux de ces amputés physiquement d'une moitié d'eux-mêmes ", leur reprochant " de vivre encore " alors qu'elle-même avait perdu " une moitié d'elle-même » (p. 1259) de sorte qu'elle était, symboliquement, devenue une femme-tronc.

Et donc une victime de la guerre, elle aussi. Puisqu'après " trois années de bonheur orgiaque » (p. 1267), "elle s'était muée en un personnage d'une

3. Les «murs badigeonnés d'un rose presque décoloré par l'humidité marine " (p. 1271) font ainsi le lien avec le paragraphe suivant lorsqu'il est dit que « le vent gonflait les toiles rayées elles aussi d'un rose dont la pâleur était là l'effet non de l'humidité mais du soleil mangeant leur couleur jusqu'à ce que peu à peu elle n'apparût plus que comme un léger frottis sur la texture grenue du tissu " (p. 1272), renvoyant ensuite à " la liseuse recouverte de cette cretonne à petits bouquets et aux fines rayures roses" (p. 1281) que cherche désespérément à retrouver le petit Claude.

4. Claude Simon nous expliquant plus que jamais dans ce dernier texte comment il fonctionne, s'intéressant à la morphologie des mots - "poule " et "boule " (p. 1285) - " et par suite aussi sans doute d'une combinaison d'images où s'amalgamaient " (ibid.) des idées pour "évoquer tout à la fois dans [son] imagination " (ibid.) ce que la mémoire en fin de compte retiendra, analogies et correspondances liées entre elles par nécessité, notamment grâce à la publicité, " comme les Pilules Orientales ou la crème Kala Busta » (ibid.), la publicité s'invitant de plus en plus au fur et à mesure que le roman avance. 
dureté rigide " (ibid.), aussi dur, autrement dit, que la pierre ou que le monument érigé à la gloire "d'une certaine rigueur militaire et d'une inflexible conception de l'honneur ${ }^{5}$ "(ibid.). Pas étonnant alors que tout le roman tourne autour de ce monument aux morts, et qu'il ne s'agisse pas tant du cinéma Castillet d'un côté et de la mer Méditerranée de l'autre, mais, entre "les portes fermées du cinéma" (p. 1264) et "la déception des photographies " (p. 1265), entre le terminus "domiciliaire " (p. 1267) de la ligne de tramway et le terminus estival de la villa d'été, entre tout cela donc, qui résume l'existence des hommes et les apitoie sur leur sort, du monument aux morts devant lequel Claude Simon écolier passe sans arrêt, dans les deux sens du terme, au point qu'à force de ne pas s'y arrêter mais de repasser sans cesse devant, il se souvient de choses qui n'y sont pas, et s'imagine (car il s'agit bien d'une affabulation cette fois) un monument qui n'est pas tout à fait celui qui se dresse, aujourd'hui encore, au centre de Perpignan.

Il oublie ainsi, ou fait semblant de l'oublier, que l'un des trois personnages représentés pour symboliser les métiers de ces hommes partis à la guerre et qui n'en sont pas revenus, n'est pas un vigneron mais, chose plus étonnante, une vigneronne :

Monument en grès rose du pays et aussi haut qu'une maison de deux étages, élevé en bordure du jardin public et où sur un fond de marbre noir (comme une page de deuil encadrée d'un matériau précieux) s'allongeaient les interminables colonnes de noms dorés à la feuille des morts de la ville bizarrement symbolisés sous la forme de trois personnages grandeur nature taillés à grands coups de ciseau dans une pierre blanche, et où, grâce aux accessoires dont ils étaient pourvus, on pouvait reconnaître un marin pêcheur son filet sur l'épaule, un vigneron un pied sur sa pelle et un maçon la truelle à la main, les trois personnages comme seulement ébauchés (volontairement, symboliquement?), rugueux (la boue des tranchées?) et que le sculpteur (apparenté à la riche famille qui produisait un apéritif réputé) avait disposés non pas sur un rang mais selon un léger arc de cercle [...] (p. 1265).

Telle est la description du monument que fait Claude Simon. Sauf que ce monument aux morts, s'il est bien en grès rose, et s'il représente bien un marin pêcheur son filet sur l'épaule ainsi qu'un maçon la truelle à la main, ne représente pas un vigneron un pied sur sa pelle, mais une vigneronne une grappe de raisin à la main, une de ces grappes cueillies dans les « vignes cramoisies ou roses de l'automne» (p. 1283) lorsque le monument a été inau-

5. De l'enfance à l'« internat parisien » avec sa discipline « militaire " (p. 1312), Claude Simon était ainsi condamné à une vie de soldat. Condamné jusque sur son lit d'hôpital, l'infirmière qui le soigne ayant un "parler militaire " (p. 1310), se retrouvant alors dans la position de sa mère sur sa liseuse avec une bonne à son service, cette bonne qui, avec «sa légère claudication » (p. 1288), était comme l'incarnation de la mort ainsi que la représente « Breughel (ou est-ce de Jérôme Bosch?...) » (ibid.). 
guré le 2 novembre 1924. Claude Simon aurait-il assisté à cette inauguration lorsqu'il avait 11 ans? Le Tramway raconte en effet les deux dernières années de son enfance à Perpignan, l'été 1924 lorsque sa mère est encore vivante, et "l'été suivant, sauf que maman n'était plus là »(p. 1292). La fin des vacances coïncidant " avec le jour des morts "(ibid.), c'est-à-dire le $1^{\text {er }}$ novembre, était-il encore présent ou avait-il déjà regagné son collège parisien où il était désormais interne?

Claude Simon ne nous le dit pas mais je ne puis m'empêcher de penser, lorsqu'il nous rappelle que sa mémoire lui joue des tours, qu'il n'est pas si ranci que ça (le verbe rancir s'employant au figuré à propos d'un esprit qui se corrompt par l'effet du temps), et qu'il tisse au contraire la trame jusque dans ses moindres détails. Statufier la mère, puisque c'est bien de l'héritière des vignobles Denamiel qu'il s'agit derrière le brouillard de la mémoire, de cette femme comparée dans tout le roman à un soldat, mais à un soldat fatigué de lutter (p. 1288) et qui semblait n'attendre que l'inauguration de ce monument pour, quelques mois plus tard, le 5 mai 1925, mourir à son tour, ne serait-ce pas la dernière étape non pas tant pour mettre la mère à la hauteur du reste de la famille - ainsi " la gracieuse tête sculptée " (p. 1294) du "général d'Intendance " (p. 1286), ainsi «le monumental buste de marbre " de l'aïeul «Conventionnel et régicide» (ibid.) - mais pour la réunir avec celui qu'elle avait épousé, contre l'avis de sa propre mère, par amour (p. 1259). Qu'elle repose enfin avec le père dont le nom était gravé sur le mur d'un de ces 30000 monuments érigés de 1918 à 1925 en France.

Sauf, une fois encore, que le nom du père n'est nulle part inscrit, ni dans les interminables colonnes de noms dorés à la feuille sur le monument aux morts de Perpignan, ni dans n'importe quel autre village de France. Comme Spinoza, le père se trouve sans sépulture à son nom. Et bien qu'il ne soit pas le seul à avoir été enseveli rapidement sur le champ de bataille dans une tombe commune et anonyme après qu'on ait récupéré sa montre pour l'envoyer à sa veuve, Antoine Louis Eugène Simon est également un soldat qui se retrouve sans monument aux morts. C'est un fait extraordinaire, en effet, de constater que Les Planches-près-Arbois, village natal de la figure paternelle, est l'un des seuls villages de France à ne pas en avoir. L'exception est remarquable et Claude Simon semble se faire un devoir de réparer l'oubli en offrant à son père et à sa mère, pour les réunir enfin en un même lieu, la plus belle des pierres tombales. 
Car après tant d'illustres aïeuls statufiés et honorés sur les monuments publics, la tradition s'arrête là. Ni le père ni la mère n'auront droit à leur buste de marbre ou à leur plaque de cuivre. À peine reste-t-il une photo à la surface de laquelle glissent quelques larmes (p. 1315). Rien d'autre. Aussi Claude Simon répare-t-il l'injure faite à ses parents en décidant, grâce à la fiction, de réunir dans un seul et même personnage de pierre deux êtres qu'il aura peu connus mais dont son œuvre se sera abondamment nourrie. Et, grâce au soi-disant brouillard de la mémoire, se l'imaginer non plus tronquée, mais "grandeur nature" (p. 1265) et comme discutant avec les deux autres personnages. Puisque sont finalement aussi décevantes les "promesses " (p. 1264) du cinéma que les photographies " figées et privées de la magie du mouvement " (Ibid.), telle est l'image ultime qu'il nous laisse, une image à fois fixe et en mouvement, pétrifiée pour l'éternité et " comme sorti[e] du royaume des morts» (p. 1265).

Sauf qu'il ne s'arrête pas là.

Car ce monument aux morts, il veut non seulement qu'on puisse le voir de loin, mais qu'il soit également une affiche publicitaire qui proclamerait : Ici gît l'œuvre de Claude Simon! Un coup de publicité génial (mais l'ironie et la dérision n'étaient pas étrangères à son auteur) auquel on arrive, bien évidemment, par le tramway, ce tram dont on ne voit d'abord, au-dessus des vignes cramoisies ou roses de l'automne, que «le panneau-réclame des Nouvelles Galeries » (p. 1283) d'un côté, et de l'autre " du cirage (où était-ce une marque de pâtes alimentaires? - je ne sais plus) Éclipse " (p. 1290); ce qu'il sait par contre, ce dont il se souvient avec précision étant ces autres publicités que l'on pouvait voir " peintes en divers endroits réservés en ville ou ailleurs, sans le moindre respect des monuments ou des édifices sur lesquels elles s'étalaient, les plus fréquentes étant celles de "SUZE - Apéritif à la gentiane", le nom SUZE en lettres monumentales jaunes au relief figuré en noir sur fond vert olive, ce qui, le temps et la patine aidant, atténuant l'éclat criard des couleurs primitives, s'accordait assez plaisamment dans une grisaille uniforme, et concurremment BYRRH, en lettres tout aussi monumentales mais blanches (et également en faux relief) sur fond rose» (p. 1290), ce rose qui est non seulement le fond du problème ${ }^{6}$, mais qui de surcroît mène tout droit aux noms

6. Voir le rose du pied du marin (p. 1284) ou celui des vignes en automne (p. 1283), le rose qui va du pied à la vigne encore lorsqu'il est question des charrettes utilisées pour les vendanges et dont les roues étaient "peintes en rose " (p. 1294) comme était «bosselé et bordé d’ocre rose " (p. 1296) le pied à demi écrasé par une comporte de 100 kilos amenée par ces mêmes charrettes. 
de SUZE et de BYRRH qui dissimulent les édifices de la ville et semblent cacher jusqu'au monument aux morts lui-même.

Et c'est précisément en juxtaposant ce monument aux morts à des panneaux publicitaires tout aussi monumentaux que Claude Simon redéfinit les relations ambigües et corrompues que l'art et la guerre entretiennent. De fait, le "sculpteur apparenté à la riche famille qui produisait un apéritif réputé " (p. 1265) du monument aux morts que décrit Claude Simon n'est autre que Gustave Violet, mort à Perpignan en 1952, qui faisait partie du groupe des Artistes roussillonnais avec l'écrivain Louis Codet, cousin de la mère de Claude Simon, tué en 1914 comme le père de ce dernier, et dont la famille, depuis 1866, produisait le célèbre Byrrh, réputé, comme la Suze, autant pour ses "vertus médicinales que gustatives ", et qui, servi "par une politique de promotion qui demeure une référence (les célèbres concours d'affiches Byrrh, entre autres) ${ }^{7}$ ", permit à la marque de connaître un développement extraordinaire. Et là encore, comme nous l'avons fait pour Spinoza, il faudrait revenir sur ce nom, Violet, autre nuance de rose et dont il est fait mention ailleurs dans le roman lorsque la date au dos de la photographie du père et de la mère jouant au tennis en juillet 1914 est écrite avec une " encre d'un vilain violet» (p. 1315).

Mais continuons plutôt avec un petit verre de SUZE que Picasso déguste en $1912^{8}$. Car en ce qui concerne l'origine de ce nom, elle n'est pas claire. Vient-il de Suzanne Jaspart, belle-sœur de son inventeur français, Fernand Moureaux, ou du cours d'eau la Suze, en contrebas du Pré-aux-Bœufs, lieu-dit non loin de Sonvilier en Suisse? Si le nom vient de Suzanne, c'est que la Suze est française. Si le nom vient de la Suze, rivière qui se jette, après un parcours de 45 kilomètres, dans le canal de la Thielle et le lac de Bienne en traversant le Vallon de Saint-Imier dans le massif du Jura, la Suze est suisse9. Mais que nous importe après tout que les deux pays se disputent la paternité du célèbre apéritif puisque Sonvilier et Les Planches-près-Arbois, village d'origine de la famille paternelle de Claude Simon où habitaient ses tantes Eugénie, Louise et Arthémise, ne sont éloignés que d'une centaine de kilomètres et que ces

7. G. Durbet, La Belle Époque dans les Pyrénées-Orientales, Association culturelle de Villefranche-deConflent, Perpignan, 2001, p. 90-92.

8. En 1912, Picasso réalise un collage intitulé "Verre et bouteille de Suze ».

9. Pour les anciens de la région, c'est un herboriste du village de Sonvilier, Hans Kappeler, qui a mis au point la formule. La boisson ne s'appelait pas encore Suze mais "Or des Alpes ", une sorte de potion que Hans Kappeler vendait dans les fermes de la région avec d'autres eaux-de-vie. Ruiné et malade, ce distillateur aurait alors été contraint de vendre sa formule à un négociant français, Fernand Moureaux. 
deux villages, séparés par la frontière franco-suisse, appartiennent à la même région comme si de la Suze paternelle au vin maternel, il n'y avait qu'un pas d'Orion. Et un pas franchi, bien évidemment, grâce au prénom de la mère : Suzanne. La Suze d'un côté, et l'âne de l'autre, cet âne " planté en travers " (p. 1289) du chemin, " aussi immobile que s'il avait été en pierre " (ibid.) et que la bonne, bras droit de la mère et incarnation de la mort, qui claudique comme le Diable (p. 1288), au visage " comme taillé dans une matière inerte, jaune" (ibid.), est seule à pouvoir faire bouger dans cet endroit où il y avait "seulement des pierres" (p. 1289).

Immortelle, inhumaine, le contraire de la gisante blonde que Claude Simon voit disparaître impuissant dans le couloir de l'hôpital, "c'était comme si maman et elle formaient une sorte de couple indissociable lié par une connaissance commune du malheur " (p. 1288) ou par " une sorte de pacte ou de lien occulte, de silencieuse connivence, comme on ne savait quelle alliance scellée du fond des âges, plus forte que le temps et la mort » (p 1299). Plus fort que le temps et la mort, en effet, cet être de pierre et de la même "dureté rigide" (p. 1267) que la mère, comme si la mère, telle une Méduse allongée sur une liseuse recouverte de "cretonne à petits bouquets et aux fines rayures roses " (ibid.), avait ce pouvoir de transformer en pierre tout son entourage avant de devenir pierre elle-même, et jusqu'au texte luimême par conséquent, un texte gravé dans la pierre puisque la pierre est sans doute la chose qui, entre photographie et cinéma, résiste le mieux au temps, un roman pour arrêter la corruption et le pourrissement des corps, un roman pour graver en lettres d'or le nom du père, statufier la mère et élever l'œuvre au rang de monument. 